\title{
DIRECT COMPUTATIONAL ALGORITHM FOR SOLVING SYSTEMS OF FREDHOLM INTEGRO- DIFFERENTIAL EQUATIONS
}

\author{
Falade Kazeem Iyanda, Mustaphar Muhammad \\ Department of Mathematics, Faculty of Computing and Mathematical Sciences, \\ Kano University of Science and Technology, P.M.B 3244 Wudil Kano State, Nigeria
}

\begin{abstract}
In this paper, we present an efficient direct computational algorithm for the numerical solution of system of Fredholm integrodifferential equations of the second kind. The proposed algorithm was based on direct computational technique of solving Fredholm integrodifferential equations. This approach is simple and computationally very attractive. Finally, illustrative examples and also the application of the proposed method to first order system of Fredholm integrodifferential show the validity and applicability of the technique.
\end{abstract}

Keywords-First order systems of Fredholm integrodifferential equations, Direct computational approach, Algorithm.

\section{INTRODUCTION}

Mathematical modeling of real-life phenomena usually results in integral and integro-differential equations (IDE), these equations arise in engineering, biological models and chemical kinetics (Kythe and Puri 2002). Integro-differential equations is an equation that the unknown function appears under the sign of integration and it also contains the derivatives of the unknown function. It can be classified into Fredholm equations and Volterra equations. The upper bound of the region for integral part of Volterra type is variable while it is a fixed number for that of Fredholm type. Fredholm integrodiferential equations play an important role in many fields such as applied mathematics: engineering, economics, modeling science, physical chemistry and oscillation theory and airfoil theory. However, in this paper, we focus on system of Fredholm integrodifferential of the form:

$$
\left\{\begin{array}{c}
u^{(i)}(x)=g_{1}(x)+\int_{a}^{b}\left(K_{1}(x, t) u(t)+\widehat{K_{1}}(x, t) v(t)\right) d t \\
v^{(i)}(x)=g_{2}(x)+\int_{a}^{b}\left(K_{2}(x, t) u(t)+\widehat{K_{2}}(x, t) v(t)\right) d t \\
x, t \in[a, b]
\end{array}\right.
$$

Subject to initial conditions

$$
\left\{\begin{array}{l}
u\left(x_{0}\right)=\tau_{1} \\
v\left(x_{0}\right)=\tau_{2}
\end{array}\right.
$$

Where $\mathrm{g}_{1}(\mathrm{x})$ and $\mathrm{g}_{2}(\mathrm{x})$ are known functions, $\tau_{1}, \tau_{2}$ are constants, $\mathrm{K}_{1}(\mathrm{x}, \mathrm{t}), \widehat{\mathrm{K}_{1}}(\mathrm{x}, \mathrm{t}), \mathrm{K}_{2}(\mathrm{x}, \mathrm{t}), \widehat{\mathrm{K}_{2}}(\mathrm{x}, \mathrm{t}) \quad$ are known kernels and $\mathrm{u}(\mathrm{x}), v(x)$ are unknown that must be determined. The theory and application of integro-differential equations are important roles in engineering and applied sciences. Thus, numerous works have been focusing on the development of more advanced and efficient methods for solving integrodiferential equations such as, (Atkinson 2011) proposed numerical approach of integral equations of the Second Kind, (Ayad 2011) proposed spline approximation for first order Fredholm Integro-differential equation, (Brunner 2004) applied Collocation method for volterra integral and related functional equations, ( Lakestani et al., 2006) proposed and applied semi-orthogonal spline wavelets approximation for Fredholm Integro -differential equations. Also in (Brunner 2004; Rabbani et al., 2007) some of results about solving Volterra integral equations are presented. Semi orthogonal spline wavelets and spline are used for solving integrodifferential equation respectively in (Ayad 1996), numerical solution of first-order linear Fredholm integro-differential equations using conjugate gradient method in (Elayaraja 2009; Dehghan 2008) proposed Chebyshev finite difference method for Fredholm integro-diferential equation,(Lackiewicz et al., 2006) Numerical solution of a Fredholm integro-diferential equation modelling neural network and in (Lakestani et al., 2006) Semi orthogonal spline wavelets approximation for Fredholm integrodiferential equations was developed.

The purpose of this paper is to employ the direct computational method discussed in (Wazwaz 2011) and formulate a suitable algorithm for the numerical solution of system of Fredholm integro-differential equations. Consequently, the algorithms was tested for four examples and results show that the formulated approach was easy, accurate and rapidly converges to the exact solution. 


\section{International Journal of Engineering Applied Sciences and Technology, 2020 \\ Vol. 5, Issue 1, ISSN No. 2455-2143, Pages 30-35 \\ Published Online May 2020 in IJEAST (http://www.ijeast.com)}

\section{DIRECT COMPUTATION METHOD (DCM)}

The DCM is a traditional method that is commonly used to handle many Fredholm integral equations (Delves 1974; Kanwal 1971). The DCM transform a FIDE to an ordinary differential equation (ODE). Then the solution of the obtained ODE is transformed to an algebraic system of equations. By calculating the solutions of the algebraic system of equations and substituting into the solution of the ODE.

Consider equation (1) and define the kernel function as follow

$$
\left\{\begin{array}{l}
\mathrm{K}_{1}(\mathrm{x}, \mathrm{t})=\sum_{k=1}^{n} f_{k}(x) m_{k}(t) \\
\widehat{\mathrm{K}_{1}}(\mathrm{x}, \mathrm{t})=\sum_{k=1}^{n} \widehat{\mathrm{f}_{\mathrm{k}}}(x) \widehat{\mathrm{m}_{\mathrm{k}}}(t) \\
\mathrm{K}_{2}(\mathrm{x}, \mathrm{t})=\sum_{k=1}^{n} e_{k}(x) h_{k}(t) \\
\widehat{\mathrm{K}_{2}}(\mathrm{x}, \mathrm{t})=\sum_{k=1}^{n} \widehat{\mathrm{e}_{\mathrm{k}}}(x) \widehat{\mathrm{h}_{\mathrm{k}}}(t)
\end{array}\right.
$$

Substitute (3) into the system of Fredholm integro-differential equations (1) to obtain

$$
\left\{\begin{aligned}
u^{(i)}(x)= & g_{1}(x)+\sum_{k=1}^{n} f_{k}(x)+\int_{a}^{b} m_{k}(t) u(t) d t+ \\
& \sum_{k=1}^{n} \widehat{\mathrm{f}_{\mathrm{k}}}(x) \int_{a}^{b} \widehat{\mathrm{m}_{\mathrm{k}}}(t) v(t) d t \\
v^{(i)}(x)= & g_{2}(x)+\sum_{k=1}^{n} e_{k}(x) \int_{a}^{b} h_{k}(t) u(t) d t+ \\
& \sum_{k=1}^{n} \widehat{\mathrm{e}_{\mathrm{k}}}(x) \int_{a}^{b} \widehat{\mathrm{h}_{\mathrm{k}}}(t) v(t) d t
\end{aligned}\right.
$$

Integrate at the right side depends only on the variable $t$ with constant limits of integration for t. Implies that each integral is equivalent to a constant. Thus, equation (4) becomes

$$
\left\{\begin{array}{c}
u^{(i)}(x)=g_{1}(x)+\mu_{1} f_{1}(x)+\mu_{2} f_{2}(x) \\
+\cdots \mu_{n} f_{n}(x)+\gamma_{1} \widehat{\mathrm{f}_{1}}(x)+\gamma_{2} \widehat{\mathrm{f}_{2}}(x)+\cdots \gamma_{n} \widehat{\mathrm{f}_{\mathrm{n}}}(x) \\
v^{(i)}(x)=g_{2}(x)+\delta_{1} e_{1}(x)+\delta_{2} e_{2}(x) \\
+\cdots \delta_{n} e_{n}(x)+\rho_{1} \widehat{\mathrm{e}_{1}}(x)+\rho_{2} \widehat{\mathrm{e}_{2}}(x)+\cdots \rho_{n} \widehat{\mathrm{e}_{\mathrm{n}}}(x)
\end{array}\right.
$$

where

$$
\begin{cases}\mu_{i}=\int_{a}^{b} m_{i}(t) u_{i}(t) d t & 1 \leq i \leq n \\ \gamma_{i}=\int_{a}^{b} \widehat{\mathrm{m}_{2}}(x) v_{i}(t) d t & 1 \leq i \leq n \\ \delta_{i}=\int_{a}^{b} h_{i}(t) u_{i}(t) d t & 1 \leq i \leq n \\ \rho_{i}=\int_{a}^{b} \widehat{\mathrm{h}_{2}}(x) v_{i}(t) d t & 1 \leq i \leq n\end{cases}
$$

Simplify both sides of (5) $i$ times from 0 to $x$, couple with initial conditions given in (2), and substituting the resulting equations for $u(x)$ and $v(x)$ into equation (6) leads to system of algebraic equations that can be solved to determine the constants $\mu_{i} \gamma_{i}$ and $\beta_{i} \alpha_{i}$. Using the obtained numerical values of these constants into the obtained equations for $u(x)$ and $v(x)$, the solutions $u(x)$ and $v(x)$ of the system of Fredholm integro-differential equations (1) follow immediately.

\section{DIRECT COMPUTATIONAL ALGORITHM (DCA)}

In this section, we formulate four steps algorithm on MAPLE 18 Mathematical software platform using equations (3) to (6) discussed in section II

Restart:
Step 1:
$u l c:=\tau_{1} ;$
$A[1]:=g_{1}(x)+\alpha ;$
$A[2]:=\operatorname{value}($ int $A[1], x))+C[1] ;$
$A[21]:=\operatorname{eval}(A[2],[x=0])=$ ulc;
$p:=\operatorname{solve}(A[21], C[1]) ;$
$q:=\operatorname{eval}(\{p\}) ;$
$C[1]:=q[1] ;$
$A[3]:=A[2] ;$
Step 2:
vlc $:=\tau_{2} ;$
$B[1]:=g_{2}(x)+\beta ;$
$B[2]:=\operatorname{value}($ int $B[1], x))+C[2] ;$
$B[21]:=\operatorname{eval}(B[2],[x=0])=u l c ;$
$p l:=\operatorname{solve}(B[21], C[2]) ;$
$q l:=\operatorname{eval}(\{p l\}) ;$
$C[2]:=q l[1] ;$
$B[3]:=B[2] ;$
Step 3:
$A[t]:=\operatorname{eval}(A[3] .[x=t]) ;$
$B[t]:=\operatorname{eval}(B[3] .[x=t]) ;$
$U:=\operatorname{int}\left(K_{1}(x, t) * A[t]+\widehat{K}_{1}(x, t) * B[t], t=\right.$
$a \ldots b) ;$
$u l:=\operatorname{value}(U) ;$
$V:=\operatorname{int}\left(K_{2}(x, t) * A[t]+\widehat{K_{2}}(x, t) * B[t], t\right.$
$\quad=a \ldots b) ;$


Published Online May 2020 in IJEAST (http://www.ijeast.com)

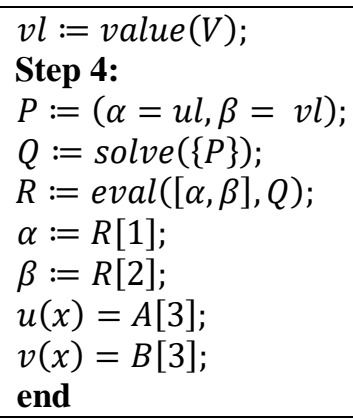

Here $\alpha, \beta, \mathrm{C}[1]$ and $\mathrm{C}[2]$ are arbitrary constants

\section{NUMERICAL EXAMPLES}

In this section we apply proposed algorithm (7) to solve system of Fredholm Integro-Differential equations. The numerical solutions obtained converge approximately to the exact solution.

\section{Example 1}

Consider the following first order system of linear Fredholm Integro-Differential equations (Wazwaz, 2011).

$$
\left\{\begin{array}{c}
u^{\prime}(x)=\cos (x)+4+\int_{0}^{\pi}(u(t)-t v(t)) d t \\
v^{\prime}(x)=-\sin (x)-\pi+\int_{0}^{\pi}(t u(t)-v(t)) d t \\
\text { subject to initial conditions } u(0)=0, v(0)
\end{array}\right.
$$

Exact solution is given

$$
\left\{\begin{array}{l}
u(x)=\sin (x) \\
v(x)=\cos (x)
\end{array}\right.
$$

Compare (7) with equation (8), we have the following:

$$
\left\{\begin{array}{c}
a=0 \\
b=\pi \\
\tau_{1}=0 \\
\tau_{2}=1 \\
g_{1}(x)=\cos (x)-4 \\
g_{2}(x)=-\sin (x)-\pi \\
K_{1}(x, t)=1 \\
\widehat{K_{1}}(x, t)=-t \\
K_{2}(x, t)=t \\
\widehat{K_{2}}(x, t)=1
\end{array}\right.
$$

Substitute the above parameters into algorithm (7), we obtain numerical solutions converge approximately to the exact solution

$$
\left\{\begin{array}{l}
u(x)=\sin (x)+\frac{129608}{3778873560001} x \\
v(x)=\cos (x)+\frac{15552000}{3778873560001} x
\end{array}\right.
$$

\section{Example 2}

Consider the following first order system of linear Fredholm Integro-Differential equations (Wazwaz, 2011)

$$
\left\{\begin{array}{c}
u^{\prime}(x)=\sin (x)+x \cos (x)+\left(2-\pi^{2}\right)+\int_{0}^{\pi}(t u(t)-v(t)) d t \\
v^{\prime}(x)=\cos (x)-x \sin (x)-3 \pi+\int_{0}^{\pi}(u(t)-t v(t)) d t \\
\text { subject to initial conditions } u(0)=0, v(0)=0
\end{array}\right.
$$

Exact solution is given

$$
\left\{\begin{array}{l}
u(x)=x \sin (x) \\
v(x)=x \cos (x)
\end{array}\right.
$$

Compare (7) with equation (11), we have the following:

$$
\left\{\begin{array}{c}
a=0 \\
b=\pi \\
\tau_{1}=0 \\
\tau_{2}=0 \\
g_{1}(x)=\sin (x)+x \cos (x)+\left(2-\pi^{2}\right) \\
g_{2}(x)=\cos (x)-x \sin (x)-3 \pi \\
K_{1}(x, t)=t \\
\widehat{K_{1}}(x, t)=-1 \\
K_{2}(x, t)=1 \\
\widehat{K_{2}}(x, t)=-t
\end{array}\right.
$$

Substitute the above parameters into algorithm (7), we obtain numerical solutions converge approximately to the exact solution.

$$
\left\{\begin{array}{l}
u(x)=x \sin (x)+\frac{7776004}{3779398439999} x \\
v(x)=x \cos (x)+\frac{64800}{3779398439999} x
\end{array}\right.
$$

\section{Example 3}

Consider the following first order system of linear Fredholm Integro-Differential equations (Wazwaz, 2011) 


$$
\begin{gathered}
u^{\prime}(x)=-\sin (x)-2 x+\frac{\pi}{2}+ \\
\int_{0}^{\frac{\pi}{2}}((x-t) u(t)+(x-t) v(t)) d t \\
v^{\prime}(x)=-\cos (x)-2 x-\frac{\pi}{2}+ \\
\left.\frac{\pi}{2}(x+t) u(t)+(x+t) v(t)\right) d t
\end{gathered}
$$

subject to initial conditions $u(0)=2, \quad v(0)=1$

Exact solution is given

$$
\left\{\begin{array}{l}
u(x)=1+\cos (x) \\
v(x)=1-\sin (x)
\end{array}\right.
$$

Compare (7) with equation (14), we have the following:

$$
\left\{\begin{array}{c}
a=0 \\
b=\frac{\pi}{2} \\
\tau_{1}=2 \\
\tau_{2}=1 \\
g_{1}(x)=-\sin (x)-2 x+\frac{\pi}{2} \\
g_{2}(x)=-\cos (x)-2 x-\frac{\pi}{2} \\
K_{1}(x, t)=(x-t) \\
\widehat{K_{1}}(x, t)=(x-t) \\
K_{2}(x, t)=(x+t) \\
\widehat{K_{2}}(x, t)=(x+t)
\end{array}\right.
$$

Substitute the above parameters into algorithm (7), we obtain numerical solutions converge approximately to the exact solution

$$
\left\{\begin{array}{l}
u(x)=1+\cos (x) \\
v(x)=1-\sin (x)
\end{array}\right.
$$

\section{Example 4}

Consider the following first order system of linear Fredholm Integro-Differential equations (Wazwaz, 2011)

$$
\left\{\begin{array}{l}
u^{\prime}(x)=-2 \sin (2 x)-\frac{\pi}{2}+\int_{0}^{\pi}((x-t) u(t)+(x+t) v(t)) d t \\
v^{\prime}(x)=2 \cos (2 x)+\frac{\pi}{2}+\int_{0}^{\pi}((x+t) u(t)+(x-t) v(t)) d t \\
\text { subject to initial conditions } \quad u(0)=1, \quad v(0)=0
\end{array}\right.
$$

Exact solution is given

$$
\left\{\begin{array}{l}
u(x)=\cos (2 x) \\
v(x)=\sin (2 x)
\end{array}\right.
$$

Compare (7) with equation (17), we have the following:

$$
\left\{\begin{array}{c}
a=0 \\
b=\pi \\
\tau_{1}=1 \\
\tau_{2}=0 \\
g_{1}(x)=-2 \sin (2 x)-\frac{\pi}{2} \\
g_{2}(x)=2 \cos (2 x)+\frac{\pi}{2}+ \\
K_{1}(x, t)=(x-t) \\
\widehat{K_{1}}(x, t)=(x+t) \\
K_{2}(x, t)=(x+t) \\
\widehat{K_{2}}(x, t)=(x-t)
\end{array}\right.
$$

Substitute the above parameters into algorithm (7), we obtain numerical solutions converge approximately to the exact solution

$$
\left\{\begin{array}{l}
u(x)=\cos (2 x) \\
v(x)=\sin (2 x)
\end{array}\right.
$$

\section{NUMERICAL RESULTS AND GRAPHS}

Table 1 Numerical solution of system of Fredholm integro-differential equation Example 1

\begin{tabular}{|c|c|c|c|c|}
\hline$x$ & $\begin{array}{c}\text { Exact } \\
\text { Solution } \\
u(x)\end{array}$ & $\begin{array}{c}\text { DMA } \\
\text { solution } \\
u(x)\end{array}$ & $\begin{array}{c}\text { Exact } \\
\text { Solution } \\
v(x)\end{array}$ & $\begin{array}{c}\text { DMA } \\
\text { solution } \\
v(x)\end{array}$ \\
\hline 0 & 1.000000 & 1.000000 & 1.000000 & 1.000000 \\
\hline 0.1 & 0.995004 & 0.995004 & 0.995004 & 0.995004 \\
\hline 0.2 & 0.980067 & 0.980067 & 0.980066 & 0.980066 \\
\hline 0.3 & 0.955338 & 0.955338 & 0.955336 & 0.955336 \\
\hline 0.4 & 0.921062 & 0.921062 & 0.921060 & 0.921060 \\
\hline 0.5 & 0.877584 & 0.877584 & 0.877582 & 0.877582 \\
\hline 0.6 & 0.825338 & 0.825338 & 0.825335 & 0.825335 \\
\hline 0.7 & 0.764845 & 0.764845 & 0.764842 & 0.764842 \\
\hline 0.8 & 0.696710 & 0.696710 & 0.696706 & 0.696706 \\
\hline 0.9 & 0.621614 & 0.621614 & 0.621609 & 0.621609 \\
\hline 1.0 & 0.540306 & 0.540306 & 0.540302 & 0.540302 \\
\hline
\end{tabular}

Table 2 Numerical solution of system of

Fredholm integro-differential equation Example 2

\begin{tabular}{|c|c|c|c|c|}
\hline$x$ & Exact & DMA & Exact & DMA \\
& Solution & solution & Solution & solution \\
& $u(x)$ & $u(x)$ & $v(x)$ & $v(x)$ \\
\hline
\end{tabular}


International Journal of Engineering Applied Sciences and Technology, 2020

Vol. 5, Issue 1, ISSN No. 2455-2143, Pages 30-35

Published Online May 2020 in IJEAST (http://www.ijeast.com)

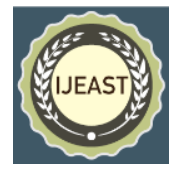

\begin{tabular}{|c|c|c|c|c|}
\hline 0 & 0.000000 & 0.000000 & 0.000000 & 0.000000 \\
\hline 0.1 & 0.099500 & 0.099500 & 0.099500 & 0.099500 \\
\hline 0.2 & 0.196013 & 0.196013 & 0.196013 & 0.196013 \\
\hline 0.3 & 0.286600 & 0.286600 & 0.286600 & 0.286600 \\
\hline 0.4 & 0.368424 & 0.368424 & 0.368424 & 0.368424 \\
\hline 0.5 & 0.438791 & 0.438791 & 0.438791 & 0.438791 \\
\hline 0.6 & 0.495201 & 0.495201 & 0.495201 & 0.495201 \\
\hline 0.7 & 0.535389 & 0.535389 & 0.535389 & 0.535389 \\
\hline 0.8 & 0.557365 & 0.557365 & 0.557365 & 0.557365 \\
\hline 0.9 & 0.559449 & 0.559449 & 0.559448 & 0.559448 \\
\hline 1.0 & 0.540302 & 0.540302 & 0.540302 & 0.540302 \\
\hline
\end{tabular}

Table 3 Numerical solution of system of

Fredholm integro-differential equation Example 3

\begin{tabular}{|c|c|c|c|c|}
\hline$x$ & $\begin{array}{c}\text { Exact } \\
\text { Solution } \\
u(x)\end{array}$ & $\begin{array}{c}\text { DMA } \\
\text { solution } \\
u(x)\end{array}$ & $\begin{array}{c}\text { Exact } \\
\text { Solution } \\
v(x)\end{array}$ & $\begin{array}{c}\text { DMA } \\
\text { solution } \\
v(x)\end{array}$ \\
\hline 0 & 2.000000 & 2.000000 & 1.000000 & 1.000000 \\
\hline 0.1 & 1.995004 & 1.995004 & 0.900166 & 0.900166 \\
\hline 0.2 & 1.980067 & 1.980067 & 0.801330 & 0.801330 \\
\hline 0.3 & 1.955336 & 1.955336 & 0.704479 & 0.704479 \\
\hline 0.4 & 1.921061 & 1.921061 & 0.610582 & 0.610582 \\
\hline 0.5 & 1.877583 & 1.877583 & 0.520574 & 0.520574 \\
\hline 0.6 & 1.825336 & 1.825336 & 0.435358 & 0.435358 \\
\hline 0.7 & 1.764842 & 1.764842 & 0.355782 & 0.355782 \\
\hline 0.8 & 1.696706 & 1.696706 & 0.282644 & 0.282644 \\
\hline 0.9 & 1.621609 & 1.621609 & 0.216673 & 0.216673 \\
\hline 1.0 & 1.540302 & 1.540302 & 0.158529 & 0.158529 \\
\hline
\end{tabular}

Table 4 Numerical solution of system of

Fredholm integro-differential equation Example 4

\begin{tabular}{|c|c|c|c|c|}
\hline$x$ & $\begin{array}{c}\text { Exact } \\
\text { Solution } \\
u(x)\end{array}$ & $\begin{array}{c}\text { DMA } \\
\text { solution } \\
u(x)\end{array}$ & $\begin{array}{c}\text { Exact } \\
\text { Solution } \\
v(x)\end{array}$ & $\begin{array}{c}\text { DMA } \\
\text { solution } \\
v(x)\end{array}$ \\
\hline 0 & 0.000000 & 0.000000 & 0.000000 & 0.000000 \\
\hline 0.1 & 0.198664 & 0.198664 & 0.198669 & 0.198669 \\
\hline 0.2 & 0.389406 & 0.389406 & 0.389418 & 0.389418 \\
\hline 0.3 & 0.564625 & 0.564625 & 0.564642 & 0.564642 \\
\hline 0.4 & 0.717333 & 0.717333 & 0.717356 & 0.717356 \\
\hline 0.5 & 0.841443 & 0.841443 & 0.841470 & 0.841470 \\
\hline 0.6 & 0.932005 & 0.932005 & 0.932039 & 0.932039 \\
\hline 0.7 & 0.985409 & 0.985409 & 0.985449 & 0.985449 \\
\hline 0.8 & 0.999528 & 0.999528 & 0.999574 & 0.999574 \\
\hline 0.9 & 0.973796 & 0.973796 & 0.973848 & 0.973848 \\
\hline 1.0 & 0.909241 & 0.909241 & 0.909297 & 0.909297 \\
\hline
\end{tabular}

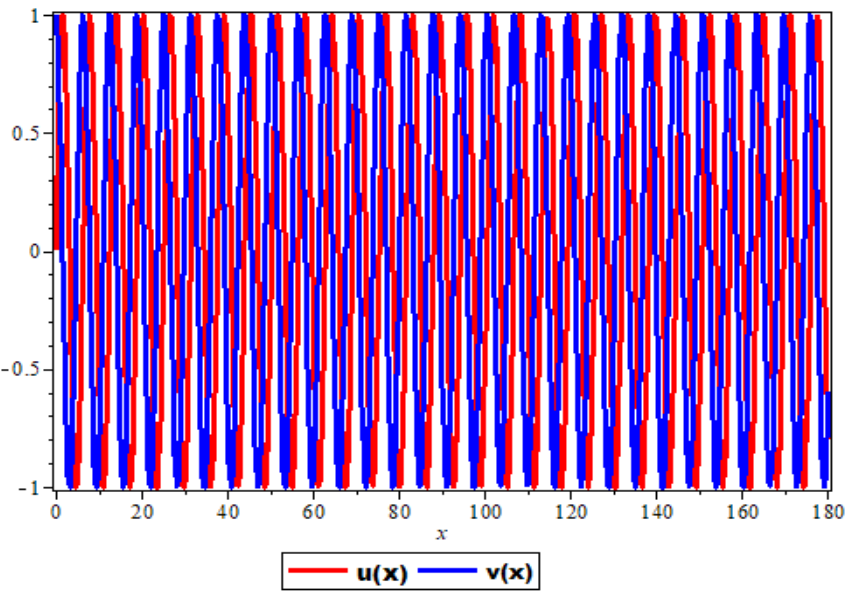

Fig. 1: Numerical solutions of $u(x)$ and $v(x)$ Example 1

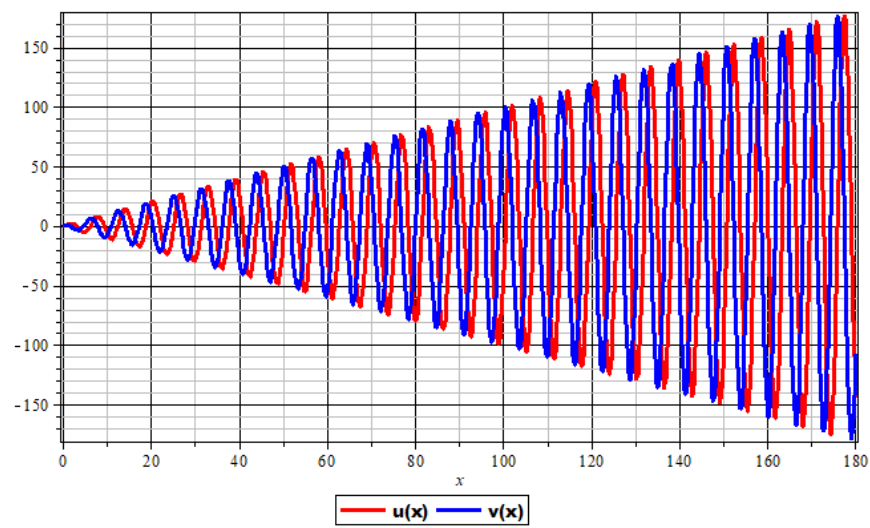

Fig. 2: Numerical solutions of $u(x)$ and $v(x)$ Example 2

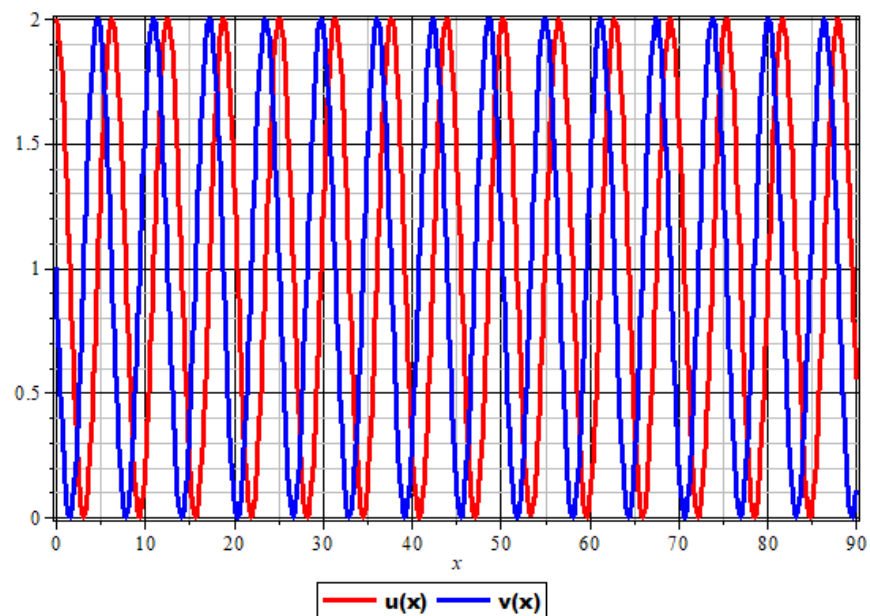

Fig. 3: Numerical solutions of $u(x)$ and $v(x)$ Example 3 
International Journal of Engineering Applied Sciences and Technology, 2020

Vol. 5, Issue 1, ISSN No. 2455-2143, Pages 30-35

Published Online May 2020 in IJEAST (http://www.ijeast.com)

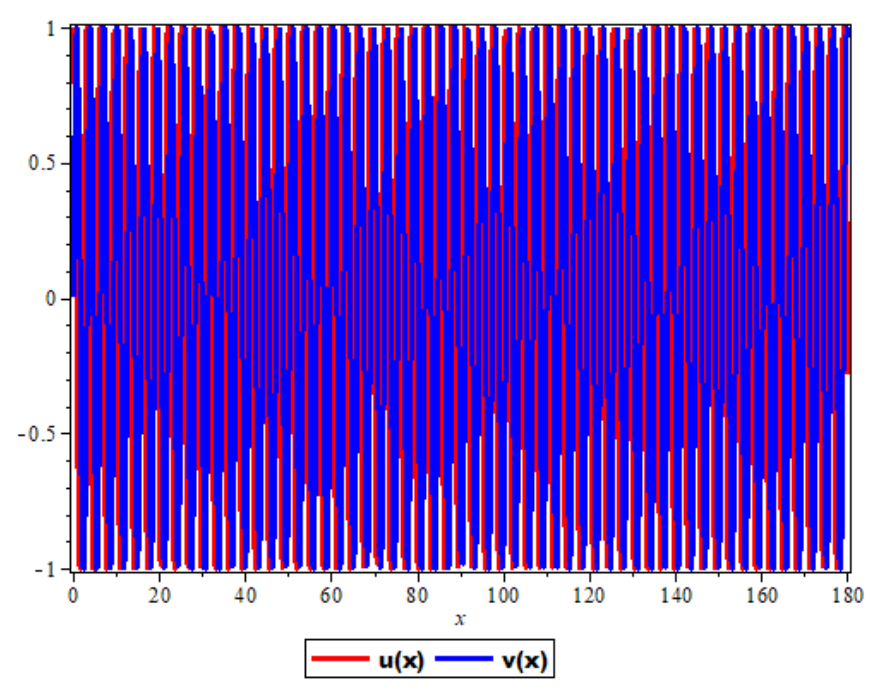

VI. CONCLUSION

In this paper, four steps algorithm was formulated using direct computational approach for the numerical solution of system of Fredholm integro-differential equations. The major benefit of this approach is to reduce the computation stress due to evaluation of differential and integration involve in integrodifferential problems and the results show that the direct computation method is a promising tool to handle this type of problems and similar problems in engineering sciences. Finally, four examples were used to demonstrate that the formulated algorithm is an efficient method to determine the solution in close form, simple and obtained results quickly. All computation works were carried out using MAPLE 18 mathematical software package.

\section{REFERENCES}

[1] Kythe P.K. and Puri P., (2002), Computational methods for linear integral equations, University of New Orlans.

[2] Atkinson, K. E.,(2011), the Numerical Solution of Integral Equations of the Second Kind. Cambridge University Press. UK, 1997. M. Rabbani and S.H. Kiasoltani/ TJMCS Vol .3 No.4 $382-389389$

[3] Ayad A. (2011) Spline Approximation for First order Fredholm Integro Differential Equation. Studia University, Babes - Bolyai, Math. 41(3), (1996), 1 - 8.

[4] Brunner H., (2004) Collocation Method for Volterra Integral and Related Functional Equations, Cambridge University Press, Combridge.

[5] Lakestani M., Razzaghi, M. and Dehghan M.(2006) Semiorthogonal Spline Wavelets Approximation for

Fredholm Integro - differential Equations. Math. Probl. Eng, 1 - 12. Article ID 96184.

[6] Rabbani M , Maleknejad K. and Aghazadeh N, (2007) Numerical computational solution of the volterra integral system of the second kind by using an expansion method, Applied Mathematics and Computation 187 1143-1146.

[7] Ayad A. (1996) Spline Approximation for First order Fredholm Integro-Differential Equation. Studia University, Babes - Bolyai, Math. 41(3), 1 - 8.

[8] Elayaraja A, Jumat S., (2009) Numerical Solution of FirstOrder Linear Fredholm Integro-Differential Equations using Conjugate Gradient Method Curtin Sarawak $1^{\text {st }}$ International Symposium on Geology (ISG1-2009) Utilizing innovative technologies for sustainable Energy Resources.

[9] Dehghan M and Saadatmandi A, (2008) Chebyshev finite difference method for Fredholm integro-diferential equation, International Journal of Computer Mathematics, vol.85, no.1, and pp.123-130.

[10] Lackiewicz Z., Rahman M. and Welfert B. D.(2006) Numerical solution of a Fredholm integro-diferential equation modelling neural networks, Applied Numerical Mathematics,vol.56,no. 3-4, pp. 423-432.

[11] Lakestani M., Razzaghi M., and Dehghan M., (2006) Semi orthogonal spline wavelets approximation for Fredholm integrodiferential equations, Mathematical Problems in Engineering, vol. 2006, Article ID 96184, 12 pages.

[12] Wazwaz A-M (2011) Linear and Nonlinear Integral Equations Methods and Applications Higher education press Beijing, pp 353- 356 April 20.

[13] Delves L.M., Walsh J.,(1974) Numerical Solution of Integral Equations, Oxford University Press, London.

[14] Kanwal R.P,(1971) Linear Integral Equations, Theory and Technique, Academic Press, New York. 